\title{
ROBUST BEAMFORMING FOR AMPLIFY-AND-FORWARD MIMO RELAY SYSTEMS BASED ON QUADRATIC MATRIX PROGRAMMING
}

\author{
Chengwen Xing, Shaodan Ma, Yik-Chung Wu and Tung-Sang Ng \\ Department of Electrical and Electronic Engineering \\ The University of Hong Kong, Hong Kong \\ Email: \{cwxing, sdma, ycwu, tsng\}@eee.hku.hk
}

\begin{abstract}
In this paper, robust transceiver design based on minimummean-square-error (MMSE) criterion for dual-hop amplifyand-forward MIMO relay systems is investigated. The channel estimation errors are modeled as Gaussian random variables, and then the effect are incorporated into the robust transceiver based on the Bayesian framework. An iterative algorithm is proposed to jointly design the precoder at the source, the forward matrix at the relay and the equalizer at the destination, and the joint design problem can be efficiently solved by quadratic matrix programming (QMP).
\end{abstract}

\section{INTRODUCTION}

Recently, amplify-and-forward (AF) MIMO relay systems have gained more and more attention from both academic and industrial communities, due to its great potential to improve the wireless channel reliability [1], [2] . For practical applications, AF MIMO relay systems are to be adopted in future communication protocols, such as Winner Project, LTE and IMT-Advanced [2], to enhance the coverage of base stations.

For transceiver design, joint LMMSE transceiver with perfect channel state information (CSI) has been investigated in [3] and an iterative algorithm has been proposed. Unfortunately, in practice, CSI is generally obtained through estimation and perfect CSI is very difficult to achieve. Robust transceiver design, which could mitigate such performance degradation by taking the channel estimation errors into account, is therefore of great importance and highly desirable for practical applications.

In this paper, we consider robust linear transceiver design for AF MIMO relay systems under imperfect CSI at both the relay and destination. The precoder at the source, the forward matrix at the relay and the equalizer at the destination are jointly designed based on minimum-mean-square-error (MMSE) criterion. With the channel estimation errors being

This work was supported by the Hong Kong Research Grants Council (Grant No. 7154/08E).

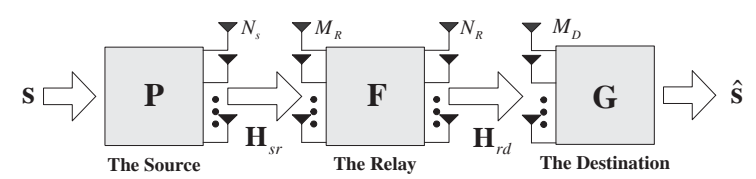

Fig. 1. Amplify-and-forward MIMO relay diagram.

modeled as Gaussian random variables, robustness is incorporated into the optimization objective function by taking expectation with respect to the channel estimation errors. The joint design problem can efficiently solved by quadratic matrix programming (QMP) [7]. Simulation results show that the proposed robust algorithm performs better than the transceiver design without taking channel estimation errors into account.

The following notations are used throughout this paper. Boldface lowercase letters denote vectors, while boldface uppercase letters denote matrices. The notations $\mathbf{Z}^{\mathrm{T}}, \mathbf{Z}^{\mathrm{H}}$ and $\mathbf{Z}^{*}$ denote the transpose, Hermitian and conjugate of the matrix $\mathbf{Z}$, respectively, and $\operatorname{Tr}(\mathbf{Z})$ is the trace of the matrix $\mathbf{Z}$. The symbol $\mathbf{I}_{M}$ denotes the $M \times M$ identity matrix, while $\mathbf{0}_{M \times N}$ denotes the $M \times N$ all zero matrix. The symbol $\mathbb{E}\{$.$\} repre-$ sents the expectation operation. The operation $\operatorname{vec}(\mathbf{Z})$ stacks the columns of the matrix $\mathbf{Z}$ into a single vector. The symbol $\otimes$ denotes the Kronecker product.

\section{SYSTEM MODEL}

In this paper, a dual-hop amplify-and-forward (AF) cooperative communication system is considered. In the considered system, there is one source with $N_{S}$ antennas, one relay with $M_{R}$ receive antennas and $N_{R}$ transmit antennas, and one destination with $M_{D}$ antennas, as shown in Fig. 1. At the first hop, the source transmits data to the relay. The received signal, $\mathbf{x}$, at the relay is

$$
\mathbf{x}=\mathbf{H}_{s r} \mathbf{P s}+\mathbf{n}_{1}
$$

where $\mathbf{s}$ is the $N \times 1$ data vector transmitted by the source with the covariance matrix $\mathbf{R}_{s}=\mathbb{E}\left\{\mathbf{s s}^{\mathrm{H}}\right\}=\mathbf{I}_{N}, \mathbf{P}$ is the precoder 
matrix with a transmit power constraint, $\operatorname{Tr}\left(\mathbf{P} \mathbf{P}^{\mathrm{H}}\right) \leq P_{s}$, with $P_{s}$ is the maximum transmit power at the source. The matrix $\mathbf{H}_{s r}$ is the MIMO channel matrix between the source and the relay. Symbol $\mathbf{n}_{1}$ is the additive Gaussian noise with covariance matrix $\mathbf{R}_{n_{1}}$.

At the relay, the received signal $\mathbf{x}$ is multiplied by a forward matrix $\mathbf{F}$, under a power constraint $\operatorname{Tr}\left(\mathbf{F} \mathbf{R}_{\mathbf{x}} \mathbf{F}^{\mathrm{H}}\right) \leq$ $P_{r}$, where $\mathbf{R}_{\mathbf{x}}=\mathbb{E}\left\{\mathbf{x x}^{\mathrm{H}}\right\}$ and $P_{r}$ is the maximum transmit power. Then the resultant signal is transmitted to the destination. The received signal $\mathbf{y}$ at the destination can be written as

$$
\mathbf{y}=\mathbf{H}_{r d} \mathbf{F} \mathbf{H}_{s r} \mathbf{P s}+\mathbf{H}_{r d} \mathbf{F} \mathbf{n}_{1}+\mathbf{n}_{2},
$$

where $\mathbf{H}_{r d}$ is the MIMO channel matrix between the relay and the destination, and $\mathbf{n}_{2}$ is the additive Gaussian noise vector at the second hop with covariance matrix $\mathbf{R}_{n_{2}}$. In order to guarantee the transmitted data $\mathbf{s}$ can be recovered at the destination, it is assumed that $N_{S}, M_{R}, N_{R}$, and $M_{D}$ are greater than or equal to $N$.

It is assumed that both the relay and destination have the estimated channel state information (CSI). When channel estimation errors are considered, we have $\mathbf{H}_{s r}=\overline{\mathbf{H}}_{s r}+\Delta \mathbf{H}_{s r}$ and $\mathbf{H}_{r d}=\overline{\mathbf{H}}_{r d}+\Delta \mathbf{H}_{r d}$, where $\overline{\mathbf{H}}_{s r}$ and $\overline{\mathbf{H}}_{r d}$ are the estimated CSI, while $\Delta \mathbf{H}_{s r}$ and $\Delta \mathbf{H}_{r d}$ are the corresponding channel estimation errors whose elements are zero mean Gaussian random variables. In general, the $M_{R} \times N_{S}$ matrix $\Delta \mathbf{H}_{s r}$ can be written as $\Delta \mathbf{H}_{s r}=\boldsymbol{\Sigma}_{s r}^{\frac{1}{2}} \mathbf{H}_{W, s r} \boldsymbol{\Psi}_{s r}^{\frac{1}{2}}$ [5], where the elements of the $M_{R} \times N_{S}$ matrix $\mathbf{H}_{W, s r}$ are independent and identically distributed (i.i.d.) Gaussian random variables with zero mean and unit variance. The $M_{R} \times M_{R}$ matrix $\boldsymbol{\Sigma}_{s r}$ and $N_{S} \times N_{S}$ matrix $\Psi_{s r}^{\mathrm{T}}$ are the row and column covariance matrices of $\Delta \mathbf{H}_{s r}$, respectively. The matrix $\Delta \mathbf{H}_{s r}$ is said to have a matrix-variate complex Gaussian distribution, which can be written as $\Delta \mathbf{H}_{s r} \sim \mathcal{C N}_{M_{R}, N_{S}}\left(\mathbf{0}_{M_{R} \times N_{S}}, \boldsymbol{\Sigma}_{s r} \otimes \boldsymbol{\Psi}_{s r}^{\mathrm{T}}\right)$ [6]. Similarly, for the estimation error in the second hop, we have $\Delta \mathbf{H}_{r d} \sim \mathcal{C} \mathcal{N}_{M_{D}, N_{R}}\left(\mathbf{0}_{M_{D} \times N_{R}}, \boldsymbol{\Sigma}_{r d} \otimes \boldsymbol{\Psi}_{r d}^{\mathrm{T}}\right)$, where the $M_{D} \times M_{D}$ matrix $\boldsymbol{\Sigma}_{r d}$ and $N_{R} \times N_{R}$ matrix $\boldsymbol{\Psi}_{r d}^{\mathrm{T}}$ are the row and column covariance matrices of $\Delta \mathbf{H}_{r d}$, respectively. It is assumed that $\mathbf{H}_{s r}$ and $\mathbf{H}_{r d}$ are estimated independently, so the channel estimation errors, $\Delta \mathbf{H}_{s r}$ and $\Delta \mathbf{H}_{r d}$, are independent.

\section{PROBLEM FORMULATION}

At the destination, a linear equalizer $\mathbf{G}$ is adopted to detect the transmitted data $\mathbf{s}$. The problem is how to design the linear precoder matrix $\mathbf{P}$ at the source, the linear forward matrix $\mathbf{F}$ at the relay and the linear equalizer $\mathbf{G}$ at the destination to minimize the mean square errors (MSE) of the received data at the destination:

$$
\begin{aligned}
& \operatorname{MSE}(\mathbf{G}, \mathbf{F}, \mathbf{P}) \\
& =\mathbb{E}\left\{\left\|\left(\mathbf{G H}_{r d} \mathbf{F} \mathbf{H}_{s r} \mathbf{P}-\mathbf{I}_{N}\right) \mathbf{s}+\mathbf{G H}_{r d} \mathbf{F} \mathbf{n}_{1}+\mathbf{G n}_{2}\right\|^{2}\right\}
\end{aligned}
$$

where the expectation is taken with respect to $\mathbf{s}, \Delta \mathbf{H}_{s r}$, $\Delta \mathbf{H}_{r d}, \mathbf{n}_{1}$ and $\mathbf{n}_{2}$. Since $\mathbf{s}, \mathbf{n}_{1}$ and $\mathbf{n}_{2}$ are independent, the
MSE expression (3) can be written as

$$
\begin{aligned}
& \operatorname{MSE}(\mathbf{G}, \mathbf{F}, \mathbf{P}) \\
& =\mathbb{E}_{\Delta \mathbf{H}_{r d}, \Delta \mathbf{H}_{s r}}\left\{\operatorname{Tr}\left(\left(\mathbf{H}_{r d} \mathbf{F} \mathbf{H}_{s r} \mathbf{P}\right)\left(\mathbf{H}_{r d} \mathbf{F} \mathbf{H}_{s r} \mathbf{P}\right)^{\mathrm{H}}\right)\right\} \\
& +\mathbb{E}_{\Delta \mathbf{H}_{r d}}\left\{\operatorname{Tr}\left(\left(\mathbf{G} \mathbf{H}_{r d} \mathbf{F}\right) \mathbf{R}_{n_{1}}\left(\mathbf{G H}_{r d} \mathbf{F}\right)^{\mathrm{H}}\right)\right\}+\operatorname{Tr}\left(\mathbf{G R}_{n_{2}} \mathbf{G}^{\mathrm{H}}\right) \\
& +\operatorname{Tr}\left(\mathbf{I}_{N}\right)-\operatorname{Tr}\left(\mathbf{G} \overline{\mathbf{H}}_{r d} \mathbf{F} \overline{\mathbf{H}}_{s r} \mathbf{P}\right)-\operatorname{Tr}\left(\left(\mathbf{G} \overline{\mathbf{H}}_{r d} \mathbf{F} \overline{\mathbf{H}}_{s r} \mathbf{P}\right)^{\mathrm{H}}\right)
\end{aligned}
$$

Because $\Delta \mathbf{H}_{s r}$ and $\Delta \mathbf{H}_{r d}$ are independent, the first term of MSE is

$$
\begin{aligned}
& \mathbb{E}_{\Delta \mathbf{H}_{s r}, \Delta \mathbf{H}_{r d}}\left\{\operatorname{Tr}\left(\left(\mathbf{G H}_{r d} \mathbf{F} \mathbf{H}_{s r} \mathbf{P}\right)\left(\mathbf{G} \mathbf{H}_{r d} \mathbf{F} \mathbf{H}_{s r} \mathbf{P}\right)^{\mathrm{H}}\right)\right\} \\
& =\operatorname{Tr}\left(\mathbf{G} \mathbb{E}_{\Delta \mathbf{H}_{r d}}\left\{\mathbf{H}_{r d} \mathbf{F} \mathbb{E}_{\Delta \mathbf{H}_{s r}}\left\{\mathbf{H}_{s r} \mathbf{P} \mathbf{P}^{\mathrm{H}} \mathbf{H}_{s r}^{\mathrm{H}}\right\} \mathbf{F}^{\mathrm{H}} \mathbf{H}_{r d}^{\mathrm{H}}\right\} \mathbf{G}^{\mathrm{H}}\right) .
\end{aligned}
$$

For the inner expectation, due to the fact that the distribution of $\Delta \mathbf{H}_{s r}$ is matrix-variate complex Gaussian with zero mean, the following equation holds [6]

$$
\begin{aligned}
\mathbb{E}_{\Delta \mathbf{H}_{r d}}\left\{\mathbf{H}_{s r} \mathbf{P} \mathbf{P}^{\mathrm{H}} \mathbf{H}_{s r}^{\mathrm{H}}\right\} & =\operatorname{Tr}\left(\mathbf{P} \mathbf{P}^{\mathrm{H}} \mathbf{\Psi}_{s r}\right) \boldsymbol{\Sigma}_{s r}+\overline{\mathbf{H}}_{s r} \mathbf{P} \mathbf{P}^{\mathrm{H}} \overline{\mathbf{H}}_{s r}^{\mathrm{H}} \\
& \triangleq \boldsymbol{\Pi}_{\mathbf{P} .}
\end{aligned}
$$

Applying (6) and the corresponding result for $\Delta \mathbf{H}_{r d}$ to (5), the first term of MSE in (4) becomes

$$
\begin{aligned}
& \operatorname{Tr}\left(\mathbf{G} \mathbb{E}_{\Delta \mathbf{H}_{r d}}\left\{\mathbf{H}_{r d} \mathbf{F} \mathbb{E}_{\Delta \mathbf{H}_{s r}}\left\{\mathbf{H}_{s r} \mathbf{P} \mathbf{P}^{\mathrm{H}} \mathbf{H}_{s r}^{\mathrm{H}}\right\} \mathbf{F}^{\mathrm{H}} \mathbf{H}_{r d}^{\mathrm{H}}\right\} \mathbf{G}^{\mathrm{H}}\right) \\
& =\operatorname{Tr}\left(\mathbf{G}\left(\operatorname{Tr}\left(\mathbf{F} \boldsymbol{\Pi}_{\mathbf{P}} \mathbf{F}^{\mathrm{H}} \mathbf{\Psi}_{r d}\right) \boldsymbol{\Sigma}_{r d}+\overline{\mathbf{H}}_{r d} \mathbf{F} \boldsymbol{\Pi}_{\mathbf{P}} \mathbf{F}^{\mathrm{H}} \overline{\mathbf{H}}_{r d}^{\mathrm{H}}\right) \mathbf{G}^{\mathrm{H}}\right) .
\end{aligned}
$$

With similar calculations applied to the second term of MSE, the total MSE in (4) can be shown to be

$$
\begin{aligned}
& \operatorname{MSE}(\mathbf{G}, \mathbf{F}, \mathbf{P})=\operatorname{Tr}\left(\mathbf{G}\left(\overline{\mathbf{H}}_{r d} \mathbf{F} \mathbf{R}_{\mathbf{x}} \mathbf{F}^{\mathrm{H}} \overline{\mathbf{H}}_{r d}^{\mathrm{H}}+\mathbf{K}\right) \mathbf{G}^{\mathrm{H}}\right)+\operatorname{Tr}\left(\mathbf{I}_{N}\right) \\
& -\operatorname{Tr}\left(\mathbf{P}^{\mathrm{H}} \overline{\mathbf{H}}_{s r}^{\mathrm{H}} \mathbf{F}^{\mathrm{H}} \overline{\mathbf{H}}_{r d}^{\mathrm{H}} \mathbf{G}^{\mathrm{H}}\right)-\operatorname{Tr}\left(\mathbf{G} \overline{\mathbf{H}}_{r d} \mathbf{F} \overline{\mathbf{H}}_{s r} \mathbf{P}\right)
\end{aligned}
$$

where $\mathbf{R}_{\mathbf{x}}=\boldsymbol{\Pi}_{\mathbf{P}}+\mathbf{R}_{n_{1}}$ and $\mathbf{K}=\operatorname{Tr}\left(\mathbf{F} \mathbf{R}_{\mathbf{x}} \mathbf{F}^{\mathrm{H}} \boldsymbol{\Psi}_{r d}\right) \boldsymbol{\Sigma}_{r d}+$ $\mathbf{R}_{n_{2}}$. Notice that the matrix $\mathbf{R}_{\mathbf{x}}$ is the autocorrelation matrix of the receive signal $\mathbf{x}$ at the relay. Finally, the joint transceiver design can be formulated as the following optimization problem

$$
\begin{aligned}
& \min _{\mathbf{G}, \mathbf{F}, \mathbf{P}} \operatorname{MSE}(\mathbf{G}, \mathbf{F}, \mathbf{P}) \\
& \text { s.t. } \operatorname{Tr}\left(\mathbf{P P}^{\mathrm{H}}\right) \leq P_{s}, \quad \operatorname{Tr}\left(\mathbf{F} \mathbf{R}_{\mathbf{x}} \mathbf{F}^{\mathrm{H}}\right) \leq P_{r} .
\end{aligned}
$$

\section{THE PROPOSED SOLUTION}

In this section, we derive an iterative algorithm to solve for $\mathbf{P}, \mathbf{F}$ and $\mathbf{G}$. In the following, it is shown that given any two variables of $\mathbf{P}, \mathbf{F}$ and $\mathbf{G}$, the remaining one can be efficiently solved. Therefore, the proposed algorithm computes $\mathbf{P}, \mathbf{F}$ and $\mathbf{G}$ iteratively, starting with initial values.

Design of $\mathbf{G}$ : When the precoder $\mathbf{P}$ at the source and the forward matrix $\mathbf{F}$ at the relay are fixed, the optimization problem (9) is an unconstrained convex optimization 
problem for $\mathbf{G}$. The optimal equalizer $\mathbf{G}$ must satisfy $\partial \operatorname{MSE}(\mathbf{G}, \mathbf{F}, \mathbf{P}) / \partial \mathbf{G}^{*}=0$ which gives

$$
\mathbf{G}=\left(\overline{\mathbf{H}}_{r d} \mathbf{F} \overline{\mathbf{H}}_{s r} \mathbf{P}\right)^{\mathrm{H}}\left(\overline{\mathbf{H}}_{r d} \mathbf{F} \mathbf{R}_{\mathbf{x}} \mathbf{F}^{\mathrm{H}} \overline{\mathbf{H}}_{r d}^{\mathrm{H}}+\mathbf{K}\right)^{-1} .
$$

Design of $\mathbf{F}$ : When $\mathbf{P}$ and $\mathbf{G}$ are fixed, the optimization problem (9) becomes

$\min _{\mathbf{F}} \operatorname{MSE}(\mathbf{G}, \mathbf{F}, \mathbf{P})$ s.t. $\operatorname{Tr}\left(\mathbf{F} \mathbf{R}_{\mathbf{x}} \mathbf{F}^{\mathrm{H}}\right) \leq P_{r}$.

Generally speaking, the optimization problem (11) is a quadratic matrix programming (QMP) problem with the variable $\mathbf{F R}_{\mathbf{x}}^{1 / 2}$ and only one constraint. We can formulate it into a semi-definite programming (SDP) problem to solve for F. However, because there is only one constraint, in the following, we introduce another algorithm to compute $\mathbf{F}$ based on Karush-Kuhn-Tucker (KKT) conditions, and has a much lower complexity. The corresponding KKT conditions of (11) are given as follows

$$
\begin{aligned}
& \mathbf{F}=\left(\overline{\mathbf{H}}_{r d}^{\mathrm{H}} \mathbf{G}^{\mathrm{H}} \mathbf{G} \overline{\mathbf{H}}_{r d}\right.\left.+\mathbf{\Psi}_{r d} \operatorname{Tr}\left(\mathbf{G} \boldsymbol{\Sigma}_{r d} \mathbf{G}^{\mathrm{H}}\right)+\lambda \mathbf{I}_{N_{R}}\right)^{-1} \\
& \times \overline{\mathbf{H}}_{r d}^{\mathrm{H}} \mathbf{G}^{\mathrm{H}} \mathbf{P}^{\mathrm{H}} \overline{\mathbf{H}}_{s r}^{\mathrm{H}} \mathbf{R}_{\mathbf{x}}^{-1} . \\
& \lambda\left(\operatorname{Tr}\left(\mathbf{F} \mathbf{R}_{\mathbf{x}} \mathbf{F}^{\mathrm{H}}\right)-P_{r}\right)=0 \\
& \lambda \geq 0, \quad \operatorname{Tr}\left(\mathbf{F} \mathbf{R}_{\mathbf{x}} \mathbf{F}^{\mathrm{H}}\right) \leq P_{r} .
\end{aligned}
$$

Obviously from (12a), in order to compute the optimal $\mathbf{F}$, the Lagrangian multiplier $\lambda$ should be calculated first. However, there is no closed-form solution of $\lambda$ simultaneously satisfying (12b) and (12c). Below we propose a low complexity method to solve (12b) and (12c). First, notice that in order to have (12b) satisfied, either $\lambda=0$ or $\operatorname{Tr}\left(\mathbf{F} \mathbf{R}_{\mathbf{x}} \mathbf{F}^{\mathrm{H}}\right)=P_{r}$ must hold. If $\lambda=0$ also makes (12c) satisfied, $\lambda=0$ is a solution to (12b) and (12c). Since given $\mathbf{G}$ and $\mathbf{P}$, the optimization problem (11) is a convex quadratic programming problem of $\mathbf{F}$, which has only one solution for $\mathbf{F}, \lambda=0$ is the only solution to (12b) and (12c) in this case.

On other hand, if $\lambda=0$ does not make (12c) satisfied, we have to solve $\operatorname{Tr}\left(\mathbf{F R}_{\mathbf{x}} \mathbf{F}^{\mathrm{H}}\right)=P_{r}$. It can be proved that when $\mathbf{G}$ and $\mathbf{P}$ are fixed, the function $\boldsymbol{f}(\lambda)=\operatorname{Tr}\left(\mathbf{F} \mathbf{R}_{\mathbf{x}} \mathbf{F}^{\mathrm{H}}\right)$ is a decreasing function of $\lambda$ which satisfies

$$
0 \leq \lambda \leq \sqrt{\operatorname{Tr}\left(\overline{\mathbf{H}}_{r d}^{\mathrm{H}} \mathbf{G}^{\mathrm{H}} \mathbf{P}^{\mathrm{H}} \overline{\mathbf{H}}_{s r}^{\mathrm{H}} \mathbf{R}_{\mathbf{x}}^{-1} \overline{\mathbf{H}}_{s r} \mathbf{P G} \overline{\mathbf{H}}_{r d}\right) / P_{r}}
$$

Due to space limitation, the proof is not presented here. Based on this result, $\lambda$ can be efficiently computed by a one-dimension search, such as bisection search or golden search. Since $\operatorname{Tr}\left(\mathbf{F} \mathbf{R}_{\mathbf{x}} \mathbf{F}^{\mathrm{H}}\right)=P_{r}$ is a stronger condition than $\operatorname{Tr}\left(\mathbf{F} \mathbf{R}_{\mathbf{x}} \mathbf{F}^{\mathrm{H}}\right) \leq P_{r},(12 \mathrm{c})$ is satisfied automatically in this case. In summary, we take $\lambda=0$, if $\boldsymbol{f}(0) \leq P_{r}$, and solve $\boldsymbol{f}(\lambda)=P_{r}$ otherwise.

Design of $\mathbf{P}$ : When $\mathbf{F}$ and $\mathbf{G}$ are fixed, after a lengthy and tedious derivation, it can be shown that the optimization problem (9) is equivalent to the following QMP problem [4]

$$
\begin{array}{ll}
\min _{\mathbf{P}} & \operatorname{Tr}\left(\mathbf{P}^{\mathrm{H}} \mathbf{A}_{0} \mathbf{P}\right)+2 \mathcal{R}\left(\operatorname{Tr}\left(\mathbf{B}_{0}^{\mathrm{H}} \mathbf{P}\right)\right)+c_{0} \\
\text { s.t. } & \operatorname{Tr}\left(\mathbf{P}^{\mathrm{H}} \mathbf{A}_{1} \mathbf{P}\right)+2 \mathcal{R}\left(\operatorname{Tr}\left(\mathbf{B}_{1}^{\mathrm{H}} \mathbf{P}\right)\right)+c_{1} \leq 0 \\
& \operatorname{Tr}\left(\mathbf{P}^{\mathrm{H}} \mathbf{A}_{2} \mathbf{P}\right)+2 \mathcal{R}\left(\operatorname{Tr}\left(\mathbf{B}_{2}^{\mathrm{H}} \mathbf{P}\right)\right)+c_{2} \leq 0,
\end{array}
$$

where the parameters are defined as follows

$$
\begin{aligned}
& \mathbf{A}_{0}=\boldsymbol{\Psi}_{s r} \operatorname{Tr}\left(\mathbf{F} \boldsymbol{\Sigma}_{s r} \mathbf{F}^{\mathrm{H}} \mathbf{M}\right)+\overline{\mathbf{H}}_{s r}^{\mathrm{H}} \mathbf{F}^{\mathrm{H}} \mathbf{M} \mathbf{F} \overline{\mathbf{H}}_{s r}, \\
& \mathbf{M} \triangleq \boldsymbol{\Psi}_{r d} \operatorname{Tr}\left(\mathbf{G} \boldsymbol{\Sigma}_{r d} \mathbf{G}^{\mathrm{H}}\right)+\overline{\mathbf{H}}_{r d}^{\mathrm{H}} \mathbf{G}^{\mathrm{H}} \mathbf{G} \overline{\mathbf{H}}_{r d}, \\
& \mathbf{B}_{0}=-\left(\mathbf{G} \overline{\mathbf{H}}_{r d} \mathbf{F} \overline{\mathbf{H}}_{s r}\right)^{\mathrm{H}}, c_{0}=\operatorname{Tr}\left(\mathbf{G}\left(\mathbf{R}_{1}+\mathbf{R}_{n_{2}}\right) \mathbf{G}^{\mathrm{H}}\right)+\operatorname{Tr}\left(\mathbf{I}_{N}\right), \\
& \mathbf{R}_{1} \triangleq \operatorname{Tr}\left(\mathbf{F} \mathbf{R}_{\mathrm{n}_{1}} \mathbf{F}^{\mathrm{H}} \boldsymbol{\Psi}_{r d}\right) \boldsymbol{\Sigma}_{r d}+\overline{\mathbf{H}}_{r d} \mathbf{F} \mathbf{R}_{n_{1}} \mathbf{F}^{\mathrm{H}} \overline{\mathbf{H}}_{r d}^{\mathrm{H}}, \\
& \mathbf{A}_{1}=\mathbf{I}_{N_{S}}, \quad \mathbf{B}_{1}=\mathbf{0}_{N_{S}, N}, \quad c_{1}=-P_{s}, \\
& \mathbf{A}_{2}=\mathbf{\Psi}_{s r} \operatorname{Tr}\left(\mathbf{F} \boldsymbol{\Sigma}_{s r} \mathbf{F}^{\mathrm{H}}\right)+\overline{\mathbf{H}}_{s r}^{\mathrm{H}} \mathbf{F}^{\mathrm{H}} \mathbf{F} \overline{\mathbf{H}}_{s r}, \\
& \mathbf{B}_{2}=\mathbf{0}_{N_{S}, N}, \quad c_{2}=\operatorname{Tr}\left(\mathbf{F} \mathbf{R}_{n_{1}} \mathbf{F}^{\mathrm{H}}\right)-P_{r} .
\end{aligned}
$$

It is known that QMP problems can be transformed into semi-definite programming (SDP) problems which can be efficiently solved by interior point polynomial algorithms [4]. Based on the properties of Kronecker product and the following definition

$$
\boldsymbol{\Omega}_{i} \triangleq\left[\begin{array}{cc}
\mathbf{I}_{N} \otimes \mathbf{A}_{i} & \operatorname{vec}\left(\mathbf{B}_{i}\right) \\
\operatorname{vec}^{\mathrm{H}}\left(\mathbf{B}_{i}\right) & c_{i}
\end{array}\right], \quad i=0,1,2,
$$

the optimization problem (14) is equivalent to

$$
\begin{array}{ll}
\min _{\mathbf{X}} & \operatorname{Tr}\left(\boldsymbol{\Omega}_{0} \mathbf{X}\right) \\
\text { s.t. } & \operatorname{Tr}\left(\boldsymbol{\Omega}_{1} \mathbf{X}\right) \leq 0, \quad \operatorname{Tr}\left(\boldsymbol{\Omega}_{2} \mathbf{X}\right) \leq 0 \\
& \mathbf{X}=\left[\operatorname{vec}^{\mathrm{T}}(\mathbf{P}) 1\right]^{\mathrm{T}}\left[\operatorname{vec}^{\mathrm{H}}(\mathbf{P}) 1\right]
\end{array}
$$

If the constraint $\operatorname{Rank}(\mathbf{X})=1$ is relaxed (it is a well-known semi-definite relaxation (SDR) [7], [8]), we have the following SDP relaxation problem

$$
\begin{array}{ll}
\min _{\mathbf{Z}} & \operatorname{Tr}\left(\boldsymbol{\Omega}_{0} \mathbf{Z}\right) \\
\text { s.t. } & \operatorname{Tr}\left(\boldsymbol{\Omega}_{1} \mathbf{Z}\right) \leq 0, \quad \operatorname{Tr}\left(\boldsymbol{\Omega}_{2} \mathbf{Z}\right) \leq 0 \\
& {[\mathbf{Z}]_{N N_{s}+1, N N_{s}+1}=1, \quad \mathbf{Z} \succeq 0,}
\end{array}
$$

where $\mathbf{Z}$ is a Hermitian matrix. Because the QMP problem (14) is a convex quadratic programming problem, the relaxation gap of SDR is zero. In other words, the optimization problems (17) and (18) have the same optimal solution [4], [9].

Summary and Convergence Analysis: Initialize $\mathbf{P}$ and $\mathbf{F}$ which satisfy $\operatorname{Tr}\left(\mathbf{P} \mathbf{P}^{\mathrm{H}}\right)=P_{s}$ and $\operatorname{Tr}\left(\mathbf{F} \mathbf{R}_{\mathbf{x}} \mathbf{F}^{\mathrm{H}}\right)=P_{r}$. For simplicity, we can take $\mathbf{P} \propto \mathbf{I}$ and $\mathbf{F} \propto \mathbf{I}$. Then the proposed iterative algorithm proceeds between (10), (12a) and (18), until $\left\|\mathrm{MSE}_{i}-\mathrm{MSE}_{i-1}\right\| \leq t$ where $\mathrm{MSE}_{i}$ is the MSE (8) in the $i$ th iteration, and $t$ is a threshold. Since for any two of the $\mathbf{P}, \mathbf{F}$ and $\mathbf{G}$ fixed, the optimization problem (9) is a convex problem for the remaining variable, the proposed algorithm is an alternative projection algorithm which is guaranteed to converges.

\section{SIMULATION RESULTS AND DISCUSSIONS}

In this section, we will investigate the performance of the proposed algorithm and for the purpose of comparison, the algorithm based on the estimated channel only (without taking 
the channel errors into account) [3] is also simulated. In order to solve the SDP problem, the matlab toolbox CVX is used [10]. In the following, we consider an AF MIMO relay system where the source, relay and destination are equipped with same number of antennas, i.e., $N_{S}=M_{R}=N_{R}=M_{D}=4$. The estimated channels $\mathbf{H}_{s r}$ and $\mathbf{H}_{r d}$ are randomly generated as

$$
\begin{aligned}
\overline{\mathbf{H}}_{s r} & =\left[\begin{array}{cccc}
1.02+.82 i & -.01-0.61 i & .12-.26 i & .02+.64 i \\
.08+.90 i & .70-1.22 i & .06+.19 i & .46+.62 i \\
1.43-1.23 i & .71-.70 i & -.23+.81 i & .03+.25 i \\
.43-.71 i & 1.56-.23 i & .29+1.30 i & -.63+.73 i
\end{array}\right], \\
\overline{\mathbf{H}}_{r d} & =\left[\begin{array}{cccc}
1.01-1.22 i & .36-.29 i & .08+.50 i & -.01+.37 i \\
.89-1.23 i & 1.05-.06 i & .32-.21 i & .45+.73 i \\
-.50+.23 i & -.45-.14 i & -.55+.42 i & 1.01+.23 i \\
-1.00+.38 i & -.54+.31 i & -.00+0.62 i & .82+1.32 i
\end{array}\right],
\end{aligned}
$$

Here the channel estimation algorithm in [5] is adopted, the correlation matrices of channel estimation errors are in the form [5].

$$
\begin{aligned}
& \boldsymbol{\Psi}_{s r}=\mathbf{R}_{T, s r}, \quad \boldsymbol{\Sigma}_{s r}=\sigma_{e}^{2}\left(\mathbf{I}_{M_{R}}+\sigma_{e}^{2} \mathbf{R}_{R, s r}^{-1}\right)^{-1}, \\
& \boldsymbol{\Psi}_{r d}=\mathbf{R}_{T, r d}, \quad \boldsymbol{\Sigma}_{r d}=\sigma_{e}^{2}\left(\mathbf{I}_{M_{D}}+\sigma_{e}^{2} \mathbf{R}_{R, r d}^{-1}\right)^{-1},
\end{aligned}
$$

where $\sigma_{e}^{2}$ denotes the estimation error variance [5]. The matrices $\mathbf{R}_{T, s r}$ and $\mathbf{R}_{R, s r}$ are the transmit and receive correlation matrices in the first hop, respectively, and similar definitions apply to $\mathbf{R}_{T, r d}$ and $\mathbf{R}_{R, r d}$ for the second hop. The widely used exponential model [5] is chosen for the transmit and receive channel correlation matrices, i.e., $\left(\mathbf{R}_{T, r d}\right)_{i, j}=$ $\left(\mathbf{R}_{T, s r}\right)_{i, j}=\alpha^{|i-j|},\left(\mathbf{R}_{R, r d}\right)_{i, j}=\left(\mathbf{R}_{R, s r}\right)_{i, j}=\beta^{|i-j|}$ where $\alpha$ and $\beta$ are the correlation coefficients.

We define the signal-to-noise ratio for the source-relay link $\left(\mathrm{SNR}_{s r}\right)$ as $\mathrm{E}_{s} / \mathrm{N}_{1}=P_{s} / \operatorname{Tr}\left(\mathbf{R}_{n_{1}}\right)$, and is fixed as $\mathrm{E}_{s} / \mathrm{N}_{1}=30 \mathrm{~dB}$. At the source, four independent data streams are transmitted. For each data stream, $10^{5}$ independent QPSK symbols are transmitted. The SNR for the relay-destination link $\left(\mathrm{SNR}_{r d}\right)$ is defined as $\mathrm{E}_{r} / \mathrm{N}_{2}=P_{r} / \operatorname{Tr}\left(\mathbf{R}_{n_{2}}\right)$. Each point in the following figure is an average of 1000 independent realization of estimation errors.

Fig. 2 shows the bit-error-rate (BER) performance of the proposed algorithm and the algorithm based on estimated channels only with different $\sigma_{e}^{2}$, when $\alpha=0.5$ and $\beta=0.4$. It can be seen that when the channel estimation errors decreases, the performances of both algorithms improve and they coincide at $\sigma_{e}^{2}=0$. Furthermore, the performance of the proposed algorithm is always better than that of the algorithm based on estimated channels only.

\section{CONCLUSIONS}

In this paper, based on the Bayesian framework, robust linear transceiver design for dual-hop AF MIMO relay systems has been considered. The precoder matrix at the source, the linear forward matrix at the relay and the linear equalizer at the destination have been jointly designed based on minimummean-square-error (MMSE) criterion. An iterative algorithm is proposed, and at each step, the design problem can be formulated as a QMP problem which can be efficiently solved.

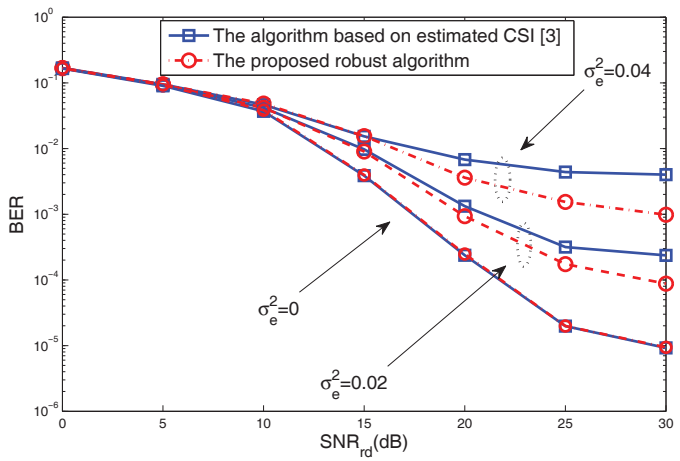

Fig. 2. The BERs for the proposed iterative algorithm and the algorithm based on estimated channels only for different $\sigma_{e}^{2}$, when $\alpha=0.5$ and $\beta=0.4$.

Simulation results showed that the performance of the proposed robust algorithm is always better than that of the algorithm based on estimated channels only.

\section{REFERENCES}

[1] C.-B. Chae, T. W. Tang, R. W. Health, and S.-Y. Cho, "MIMO relaying with linear processing for multiuser transmission in fixed relay networks," IEEE Trans. Signal Processing, vol. 56, no. 2, pp. 727-738, Feb. 2008.

[2] S. Stefania, I. Toufik, and M. backer, LTE, the UMTS Long Term Evolution: From Theory to Practice. Wiley, 2009.

[3] Y. Rong, X. Tang, and T. Hua, "A unified framework for optimizing linear non-regenerative multicarrier MIMO relay communication systems," IEEE Trans. Signal Processing, vol. 57, no. 12, pp. 4837-4851, Dec. 2009.

[4] A. Beck, "Quadratic matrix programming," SIAM Journal on Optimization, vol. 17, no. 4, pp. 1224-1238, 2007.

[5] M. Ding and S. D. Blostein, "MIMO minimum total MSE transceiver design with imperfect CSI at both ends," IEEE Trans. Signal Processing, vol. 57, no. 3, pp. 1141-1150, March 2009.

[6] A. Gupta and D. Nagar, Matrix Variate Distributions. London, U.K.: Chapman\&Hall/CRC, 2000.

[7] A. Beck, "Convexity properties associated with nonconvex quadratic matrix functions and applications to quadratic programming," Journal of Optimization Theory and Applications, 142:1-29, 2009.

[8] Y. Ye and S. Zhang, "New results on quadratic minimization," SIAM Journal on Optimization, vol. 14, no. 1, pp. 245-267, 2003.

[9] A. Beck and Y. C. Eldar, "Strong duality in nonconvex quadratic optimization with two quadratic constraints," SIAM Journal on Optimization, vol. 17, no. 3, pp. 884-860, 2006.

[10] M. Grant, S. Boyd, and Y. Y. Ye, "CVX: Matlab Software for Disciplined Convex Programming," available at: http : //www.stanford.edu/boyd/cvx/, V.1.0RC3, Feb. 2007. 